137, by about 90. The spectrum evidence confirms then the determination 225 made by Madame Curie.

As quite a number of investigators are working at relations between spectrum data and atomic weight, it seems important to make generally known the fact that mathematical series, like that of Balmer's law or that given above, are the main feature in the laws of spectra. The approximate law of Rydberg arises from the fact that the atomic weights of the elements form a series. Certain relations between this series and the series belonging to the spectra of the natural families are probably the cause of Rydberg's approximate law, which is not suitable for the extrapolation attempted by Runge and Precht with their modified form of it, unless other better means of estimating the atomic weight are lacking. All that we are warranted in saying at present is that the atomic weights of some of the elements in a family are nearly proportional to some power of $\mathrm{A}+\mathrm{B}\left(\mathrm{x}-3 n / 2+7 n^{2} / 2\right)$, where $n$ has positive integral values, and $\mathrm{A}$ and $\mathrm{B}$ are parameters characteristic of the family.

Melbourne, March.

\section{Graphic Methods in an Educational Course in Mechanics.}

I SHOULD be glad if I could, through the columns of NATURE, elicit opinions from those who have taught mechanics from the beginning as to the advisability of either omitting graphical methods altogether from an educational course in mechanics or of introducing them at a very late stage.

By graphical methods I mean those methods which depend entirely on the use of mathematical instruments of precision, and from which calculation is absent. I do not refer to the plotting of curves from results obtained analytically, or to such simple graphical considerations as enable one to draw (freehand) a useful working figure.

I myself, after many years of teaching, have come to the conclusion that until the principles of statics and dynamics have been thoroughly grasped, it is better to keep graphical methods out of sight altogether.

My contentions are as follows:-

(I) Analytical methods give a grasp of the principles of statics, while graphical methods disguise them. When a body is at rest and in equilibrium, the obvious facts are that it does not move in this direction or in that, and does not rotate. Now the idea of a resolute as the effective component of a force in any direction is one readily grasped, and the analytical statement that "the resolutes in any direction balance one another" brings vividly before the mind the equilibrium as regards translation. Any experiment made suggests this balancing of resolutes. But the closing of a polygon of forces, on the contrary, does not suggest, with anything like the same degree of vividness, that there is no translation. In fact, the closed polygon of forces, representing as it does a couple, rather suggests that there is rotation. An experiment with a body on an inclined plane, for example, suggests a balance of resolutes and does not suggest a triangle of forces.

Again, as regards rotation. The analytical method of the "balancing of moments" brings clearly before the mind the fact that the body does not rotate. I am sure that most people will agree with me when I say that the corresponding graphical proposition, that " the funicular polygon closes," will not suggest non-rotation to any ordinary learner.

(2) Analytical methods must be mastered in any case. In any educational course, it is important that the learner shall have to rely on as few principles as possible. Now when he has mastered the principles of "resolution" and "taking moments," he can be led to attack any useful problen in statics without further theory.

"But he may master the "polygon of forces" and the " funicular polygon," and yet find himself totally unable to deal with machines and with other constantly occurring cases of equilibrium. He will find that, while he can obtain by graphical methods the resultant of a system of NO. 1800 , VOL. 69]

forces if these be parallel, he will probably fail if the forces be not parallel (and non-concurrent), owing to the difficulty of getting his diagram on to a given sheet of paper. In fact, analytical methods must be mastered, while graphical methods, however convenient in certain cases, need not be mastered save for special professional purposes. If, then, there be not time for both, it is the latter that should be sacrificed. A student well trained in analytical methods can always pick up graphical methods rapidly when he needs them for special work.

(3) Analytical methods connect statics with dynamics. I do not think that this contention will be disputed. Regarded analytically, statics are a part of dynamics; the equations are the same and the ideas are the same, only the acceleration, in statics, is zero.

(4) Graphical methods confuse learners of statics. Here I rely on experience, and report what I have observed. I have noticed, over and over again, that, while a learner of analytical statics may fail to solve a problem, he yet knows what he is trying to do, and he does not, as a rule, lose sight of principles.

But I find that beginners, who have learned something of graphic statics, appear to lose sight of principles altogether, and are content to make the wildest "shots." They make triangles out of ladders, walls, and ground, and continually take the lengths of bars or strings to represent the stresses in them in their attempts to "get 2 triangle of forces."

I find no beginner so difficult to teach as one who has learned some graphic statics at a preparatory school, and I much prefer those who have learned no statics at all. There seems to be something in graphical methods that paralyses the learner's powers of thought and reasoning, or at least allows them to slumber.

To sum up. I have come to the conclusion that graphical methods (as defined above) should be reserved for a relatively late stage in any educational course in mechanics, or even be omitted altogether until required for special work. In addition to the reasons given above, I may add that graphical work consumes an amount of time that seems out of proportion to the mental training and knowledge of principles gained.

Devonport, April

W. LARDEN.

\title{
Sunspots and Temperature.
}

THE following view of this subject (related to that given by Dr. Lockyer a short time ago) may be of interest.

Consider the last five sun-spot waves, as measured from the first year after a minimum to the next minimum, thus :-

(I) $1844-56$ (I 3 years)

(2) I $857-67$ (II years)

(3) $1868-78$ (I I years)

(4) $1879-89$ (I I years)

(5) $1890-1901$ ( 12 years)

$$
\begin{array}{rrrr}
\ldots & \ldots & \text { Maximum } & \mathrm{I} 848 \\
\ldots & \ldots & , & \mathrm{I} 860 \\
\ldots & \ldots & , & \mathrm{I} 870 \\
\ldots & \ldots & , & \mathrm{I} 883
\end{array}
$$

Using Wolf and Wolfer's sun-spot numbers, and finding the annual average for each of these waves, we get the curve marked A (dotted line) in Fig. I (p. 608).

Ascertaining next the averages of several meteorological items at Greenwich for those periods, we obtain curves B, C, D, E, F. The items are :-

(B) Mean temperature of winter (December-February).

(C) Frost days in winter (an inverted curve).

(D) Days with maximum temperature $70^{\circ}$ or more (in year).

(E) Mean temperature of summer (June-August).

(F) Mean temperature of year.

The amount of agreement between these weather curves and the sun-spot curve seems remarkable. The sun-spot wave with highest average number (that for 1868-78) corresponds with the time of greatest warmth in each case, and 